\title{
Quelques aspects hydrologiques des transferts de solutés par infiltration vers les nappes : nitrates et phytosanitaires
}

\author{
Some hydrologic aspects of solutes transport by infiltration \\ towards the aquifers: nitrates and pesticides
}

\author{
par A. Landreau, \\ Service Géologique National,BRGM \\ C. Mouvet* et B. Normand, \\ Direction de la Recherche, BRGM \\ * Auteur à contacter
}

The contamination of groundwater by substances, mainly nitrates and pesticides, resulting from agricultural activities depends on three essential factors:

- the physical characteristics of the aquifer system: these characteristics play a major role in protecting the groundwater against pollutants (attenuation) and/or in postponing the outbreak of the contamination (delay);

- the intensity and mode of groundwater recharge: the infiltrating water is the transfer vector of contaminants towards the groundwater;

- the quantities of solutes available for infiltration towards the groundwater, i.e. the quantities not retained by the soil/plant/microorganism system.

The paper presents some major characteristics of the main types of aquifer in France, notably with regard to pollution vulnerability, and data on the spatial variability and quantitative importance of infiltration, a mechanism that plays a key role in the pollutant concentrations likely to be reached in the groundwater.

\section{I $\square$ RELATIONS ENTRE LES PRINCIPALES CARACTÉRISTIQUES PHYSIQUES DES SYSTÈMES AQUIFÈRES ET LES TRANSFERTS DE POLLUANTS}

De manière simplifiée, le système aquifère peut être conceptuellement représenté par trois compartiments en continuité hydraulique : le sol, la zone non-saturée et la nappe d'eau souterraine. Chacun de ces compartiments possède des caractéristiques propres, en fonction desquelles les interactions avec les polluants peuvent varier. Ces compartiments joueront chacun un rôle, soit dans l'atténuation du signal d'entrée, soit dans les délais de transfert vers la nappe, soit au niveau de la propagation dans la nappe (Fig. 1).

\subsection{Le sol : l'atténuation du signal d'entrée}

Le sol joue un rôle essentiel dans le contrôle des transferts de matière vers les nappes. Les processus de transferts d'eau et d'évolution des composés dans ce compartiment sont particulièrement bien connus, tout du moins des spécialistes agronomes et en sciences du sol. Seuls seront donc évoqués succinctement les aspects essentiels liés aux transferts vers les nappes.

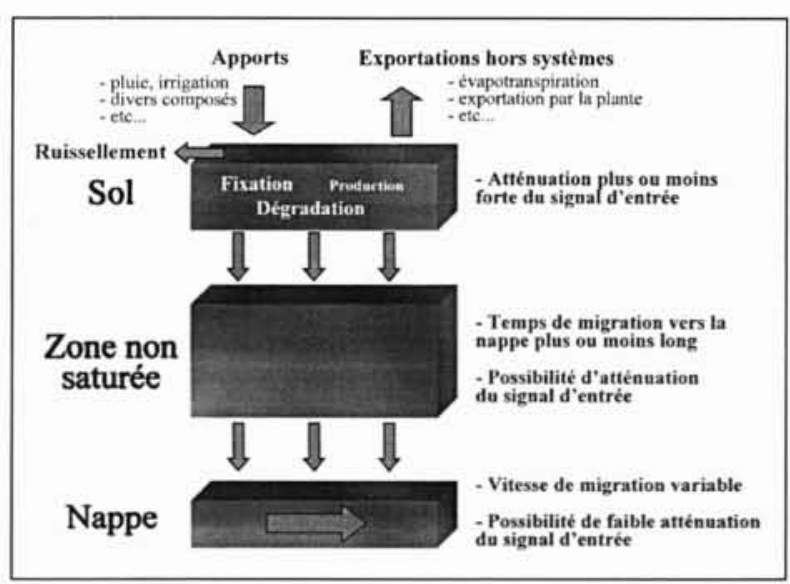

1. Schématisation du transfert de composés dans le système sol-zone non saturée-nappe.

L'utilisation, en quantité variable selon la culture, d'eau et de solutés par les plantes contribue à limiter les flux susceptibles de migrer vers les nappes ; c'est notamment le cas pour les nitrates apportés au sol comme éléments fertilisants. Toutefois, suite à un excès d'apport, ou par le biais des résidus de cultures pour les nitrates, une partie de ces éléments 
est susceptible de se retrouver disponible pour un transfert dans le système. Dans le cas des phytosanitaires, l'effet des végétaux sur les flux via la prise de solutés (de l'ordre de $10 \%$ pour l'atrazine et le maïs [1]), est moins important que pour les nitrates (de l'ordre de $65 \%$ pour le maïs [2]).

Suivant la nature du sol (granulométrie, structure, teneur en matières organiques et en argiles, teneur en eau ...), des phénomènes de fixation (adsorption) ont lieu, de manière plus ou moins intense et plus ou moins réversible suivant la nature des composés. Le système racinaire et la microflore interviennent également dans ces phénomènes de fixation. Cette potentialité de fixation de certains composés, en particulier les phytosanitaires, contribue à réduire les flux susceptibles de dégrader la qualité de l'eau des nappes. L'adsorption des phytosanitaires varie énormément selon les propriétés des molécules et selon le type de sol, notamment la teneur en carbone organique. Le $K_{o c}$, coefficient d'adsorption pondéré par la teneur en carbone organique du sol, permet de comparer la rétention potentielle de diverses molécules. Les valeurs de $K_{o c}$ étant très variables, on utilise le plus souvent le logarithme décimal, $\log K_{o c}$. L'atrazine, composé actuellement le plus fréquemment détecté dans les eaux souterraines, a un $\log K_{o c}$ entre 1,59 et 2,24 [3]. Pour l'isoproturon, les valeurs de la littérature du $\log K_{o c}$ varient entre 1,90 et 2,36 ; pour le glyphosate, la valeur de 2,22 est suggérée [3].

Le compartiment sol, via les systèmes racinaires et la flore microbienne, se caractérise également par une activité biologique propice à la transformation de certains composés. Il peut s'agir de la minéralisation de la matière organique en forme ammoniacale $-\mathrm{NH}_{4}{ }^{+}$- et nitrates $-\mathrm{NO}_{3}{ }_{-}^{-}$, ou, dans une moindre mesure, du processus inverse de réorganisation de l'azote minéral sous forme organique. Cette activité biologique est également responsable de l'apparition de métabolites des molécules mères de phytosanitaires, telle la déséthylatrazine, produit de dégradation de l'atrazine fréquemment détecté dans les eaux souterraines.

L'épaisseur du sol, sa texture et sa structure jouent également un rôle dans la limitation des transferts d'eau et de contaminants vers les compartiments sous-jacents. Des épaisseurs de sol de l'ordre de la dizaine de $\mathrm{cm}$ offrent peu de protection vis-à-vis du transfert de contaminants vers les nappes ; des sols d'épaisseur de l'ordre du mètre ou plus, avec des textures de type limoneuse, constituent des écrans plus efficaces. L'épaisseur d'un sol n'est toutefois pas un critère suffisant pour garantir une forte atténuation des flux. L'écoulement de l'eau au travers du sol est un facteur clé dans le contrôle de ces flux, notamment via des phénomènes de transferts rapides et d'écoulements préférentiels, dont l'importance potentielle au niveau des flux de phytosanitaires commence seulement à être quantifiée [4-7].

\subsection{La zone non-saturée (ZNS) : le contrôle des temps de transfert}

Par rapport au sol, siège de nombreuses interactions physico-chimiques et biologiques susceptibles d'atténuer les flux, la ZNS intervient essentiellement au niveau des temps de transfert. Les solutés véhiculés par l'eau migrent à travers cette zone selon une composante majoritairement verticale.

Suivant la structure physique de ce compartiment (plus ou moins fissuré, ou poreux) et son épaisseur, les temps de transfert vers le compartiment " nappe " peuvent varier de quelques jours à quelques dizaines d'années. A titre d'exemple, la vitesse d'infiltration peut être de l'ordre de 1 mètre par an dans des formations crayeuses peu fissurées (ex : craie de Champagne), de l'ordre de la dizaine de mètres par an dans des formations calcaires plus fissurées telles que les calcaires de Beauce, et de plusieurs mètres par heure dans des terrains karstiques.

La figure 2 illustre la vitesse de progression d'un flux de pollution en nitrates dans la ZNS de la craie en Champagne. L'évolution au cours du temps des profils de teneurs en nitrates indique, dans ce milieu et pendant la durée de l'étude, un temps de transfert de l'ordre de un mètre par an.

Au contraire du sol ou de la nappe, les moyens d'investigation de la ZNS sont limités et leur mise en œuvre est difficile. Les niveaux de contamination dans la ZNS, et leur évolution spatiale et temporelle, peuvent toutefois être étudiés par diverses techniques :

- la mise en place, à différentes profondeurs (Fig. 3) de bougies poreuses qui, mises en dépression, permettent de capter, dans certaines conditions de teneur en eau, l'eau environnant la tête poreuse. Ce dispositif est pérenne et permet, une fois installé, le suivi de transferts de solutés. Sa mise en place est toutefois délicate et n'est pas envisageable dans tous les contextes ;

- le carottage, qui permet d'échantillonner les solides et fluides de la ZNS à différentes profondeurs. Les échantillons ainsi récupérés sont ensuite traités pour extraire, par lixiviation ou centrifugation, l'eau interstitielle sur laquelle peuvent être effectuées différentes analyses ; les solides eux-mêmes

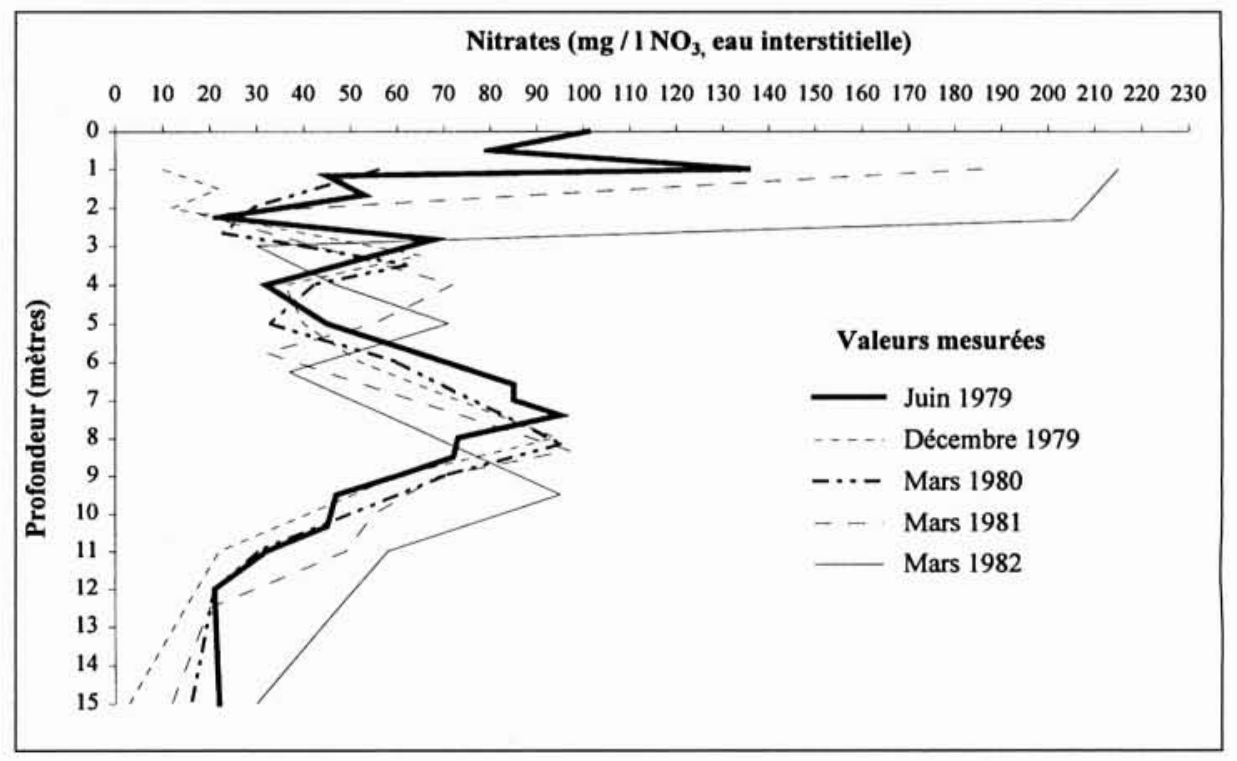

2. Suivi expérimental du transfert des nitrates dans la craie de Champagne par mesure dans l'eau interstitielle [13]. 


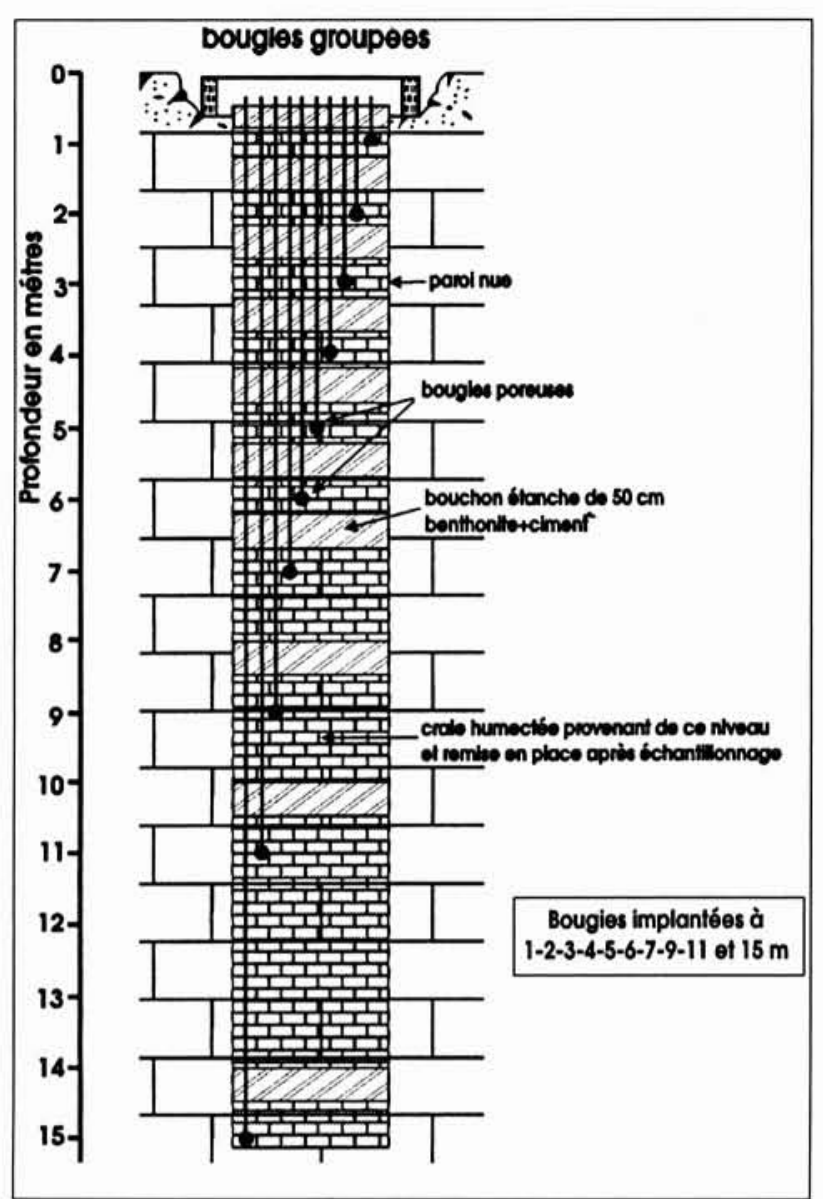

3. Dispositif de prélèvements dans la zone non saturée par bougies poreuses mis en cuvre par le BRGM dans la craie en Champagne [13].

peuvent être utilisés pour différentes déterminations utiles à la compréhension des transferts.

Outre son rôle dans le contrôle des temps de transfert, la ZNS peut également jouer un rôle dans l'atténuation des signaux. En conditions normales et au-delà de quelques mètres de profondeur, la ZNS est surtout le siège de transformations abiotiques, même si une certaine activité microbienne y est présente [8-12]. Les phénomènes de rétention dans la ZNS sont essentiellement dus à la présence, en plus ou moins grandes quantités, d'argiles, de matière organique et d'oxydes ou hydroxydes métalliques. Pour les phytosanitaires, des mesures des coefficients d'adsorption sur un profil vertical de plusieurs mètres peuvent ainsi montrer, superposées à une diminution régulière avec la profondeur, des zones où la rétention redevient plus forte (Fig. 4).

\subsection{La nappe (zone saturée) : le révélateur}

Les caractéristiques physiques du magasin aquifère interviennent essentiellement en termes de plus ou moins grande possibilité de migrations, essentiellement latérales, et de vitesse de propagation de la pollution. Les phénomènes d'atténuation du signal d'entrée ne sont le plus souvent perceptibles que sur le moyen ou le long terme. L'atténuation se note surtout en termes de concentration, par effet de dilution dans le stock d'eau de la nappe. Toutefois cette dilution n'est pas instantanée et ne se fait pas nécessairement dans l'ensemble du stock d'eau : beaucoup de nappes sont hétéro-

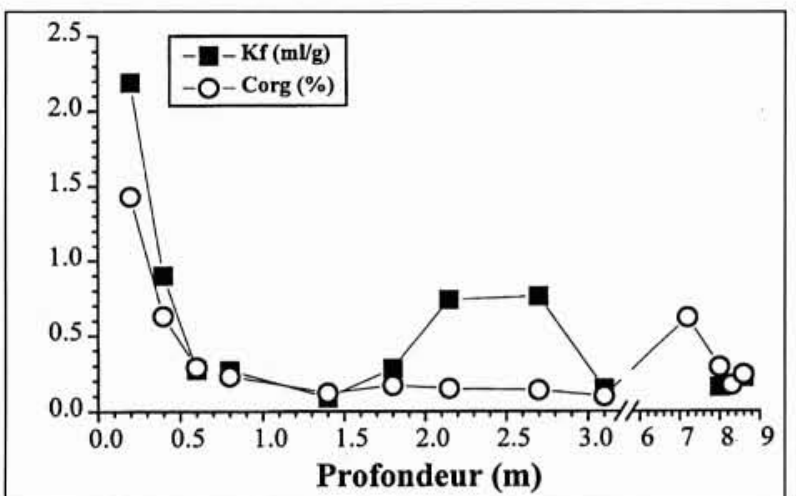

4. Evolution avec la profondeur du coefficient d'adsorption de l'atrazine et de la teneur en carbone organique des solides depuis la surface du sol jusqu'au matériau constitutif de l'aquifère (forage B22, site de Krauthausen, Allemagne).

gènes de par la nature des matériaux qui constituent l'encaissant, et la dilution s'y fait uniquement sur une partie de l'épaisseur de la nappe.

S'ils sont a priori moins intenses que dans le compartiment sol, des phénomènes physico-chimiques susceptibles de transformer ou de retenir tout ou partie du polluant peuvent néanmoins intervenir dans les nappes. Dans les nappes dites captives, les conditions réductrices du milieu sont propices à certaines transformations, telles que la dénitrification pour les nitrates. Dans le cadre d'une étude menée sur la craie du nord de la France au niveau de l'interface nappe libre-nappe captive, une baisse des teneurs en nitrates de l'ordre de $85 \%$ sur une distance d'environ $2,5 \mathrm{~km}$ a été mise en évidence (figure 5).

Les phénomènes de dénitrification observés parfois dans certains aquifères tels que la craie sont de type biochimique et de nature hétérotrophe. Des réactions entre les nitrates et d'autres composés que la matière organique peuvent toutefois conduire également à la dénitrification. Un programme d'études dans un bassin versant du massif Armoricain à forte activité d'élevage, où les concentrations en nitrates pouvant atteindre $200 \mathrm{mg} / \mathrm{l}$ dans la partie supérieure de la nappe deviennent nulles à quelques mètres de profondeur, a montré que l'aquifère schisteux est le siège d'une dénitrification intense et rapide. Les teneurs en $\mathrm{NO}_{3}{ }^{-}$sont divisées par 2 après un temps de circulation dans le schiste compris entre 2 et 8 jours [15]. Cette dénitrification est induite par 3 types de réaction : une réaction biochimique hétérotrophe faisant intervenir le carbone organique transporté avec les nitrates, une réaction biochimique autotrophe faisant intervenir les minéraux sulfurés (pyrite), et une réaction chimique faisant intervenir le fer des minéraux sulfurés et silicatés [16]. Pour les phytosanitaires, aucune étude consacrée spécifiquement à la dégradation dans les nappes ne semble avoir été publiée à l'heure actuelle.

Une certaine typologie globale des nappes en terme de vulnérabilité vis-à-vis des pollutions peut être établie en distinguant :

- les nappes " libres" de type karstique, dans lesquelles la propagation est rapide et peut intéresser de grands domaines aquifères qui se trouvent ainsi contaminés. Dans ces systèmes, l'arrêt des causes de pollution peut se manifester rapidement par une baisse du niveau de contamination. 


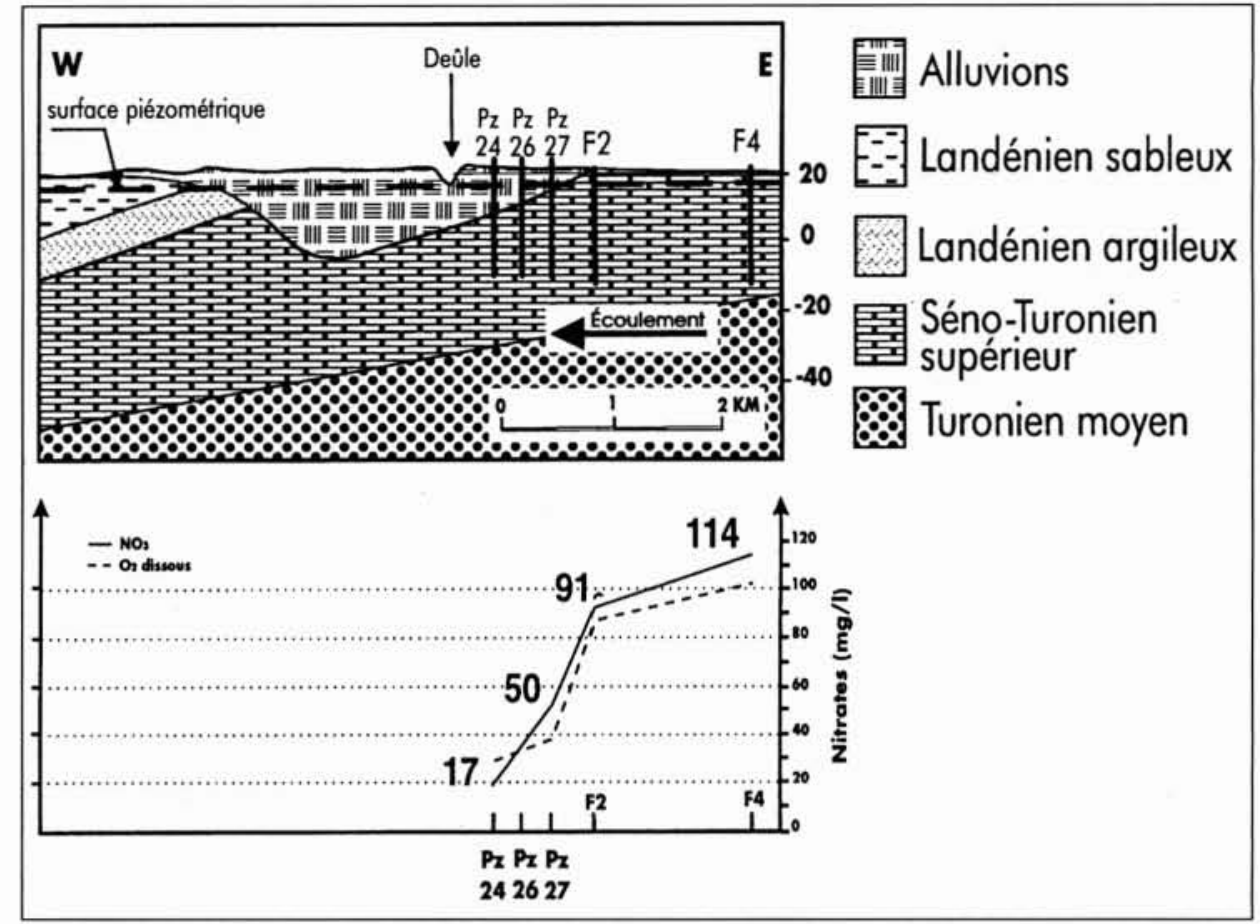

5. Mise en évidence de la dénitrification à l'interface nappe libre nappe captive sur la craie du Nord de la France [14].

- les grandes nappes " libres " à structure poreuse ou fissurée, ou mixte (cas de la craie), dans lesquelles la pollution, qui peut s'étendre sur de grandes distances, va se propager à des vitesses de quelques dizaines de mètres à quelques kilomètres par an. Dans ces milieux, l'arrêt des causes de pollution peut être sans effet tangible sur la contamination de l'eau pendant plusieurs années. La contamination va décroître généralement lentement au cours du temps, par effet de dispersion, de dilution, voire de dégradation lente du produit (cas des pesticides). A titre d'exemple, la figure 6 illustre l'évolution observée et modélisée des teneurs en nitrates dans l'eau souterraine à l'un des exutoires de la nappe des calcaires de Champigny (sources de la Voulzie). A partir d'une bonne connaissance du contexte hydrogéologique et d'une longue chronique des concentrations en nitrates observées, qui montrent une croissance très accélérée entre 1964 et 1983 , un modèle de simulation de l'évolution des teneurs a été établi et utilisé [17] pour prévoir à partir de 1983 l'évolution des teneurs selon divers scénarios, dont deux sont présentés sur la figure 6. Dans l'hypothèse d'une activité agricole inchangée sur le bassin d'alimentation des sources par rapport à celle de l'année 1983, l'augmentation des teneurs continue pour se stabiliser vers $100 \mathrm{mg} / \mathrm{l}$ au bout d'environ 50 ans. Dans l'hypothèse d'un épandage d'engrais ramené à partir de 1983 à un niveau équivalent à celui pratiqué dans les années 1960 (très faible utilisation d'engrais), la concentration se maintient à son niveau de 1983 pendant une dizaine d'années pour décroître ensuite lentement et atteindre $40 \mathrm{mg} / \mathrm{l}$ après 40 ans.

- les nappes " libres" constituant de petits systèmes aquiferes indépendants les uns des autres, en terrains sédimentaires ou de socle. Il s'agit de nappes généralement vulnérables aux pollutions ; l'étendue spatiale des éventuelles pollutions est assez limitée vu la taille de ces systèmes. Dans ce type de nappes libres, l'arrêt des causes de pollution de l'eau peut être suivi d'une amélioration assez rapide de la qualité de l'eau.

- les grandes nappes profondes dites captives, généralement bien protégées des pollutions par des couches imperméables relativement épaisses. On peut néanmoins observer dans ces nappes des contaminations localisées, dues le plus souvent à des manques de précaution en matière d'exploitation de la ressource, comme par exemple des forages mal conçus mettant en communication une nappe profonde avec une nappe libre supérieure contaminée, ou une exploitation intensive de la nappe captive près de l'interface avec la nappe libre. Cette exploitation intensive peut avoir pour effet de déplacer la zone de contamination de la nappe libre audelà de la limite de captivité. Toutefois, dans le cas des nitrates, des phénomènes de dénitrification, très souvent observés, limitent l'extension de cette contamination dans la partie captive (figure 5).

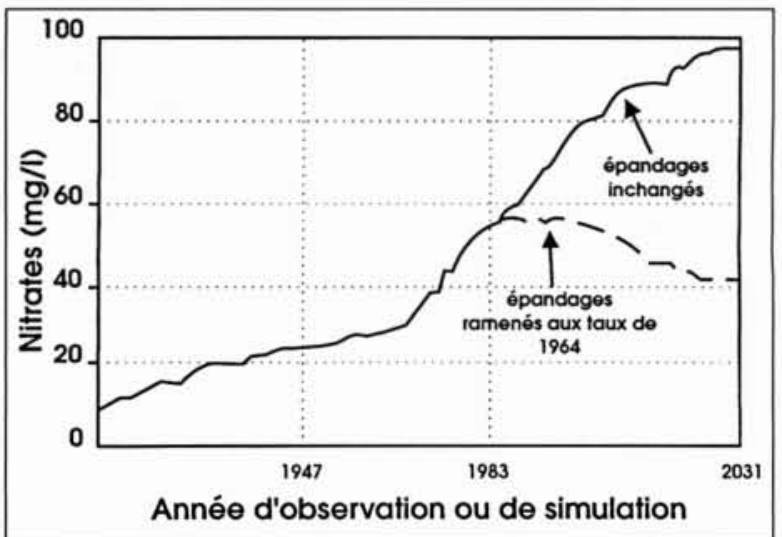

6. Sources de la Voulzie. Simulation à partir du modèle BICHE de l'évolution des teneurs en nitrates après 1983 selon deux scénarios de pratiques agricoles [17]. 


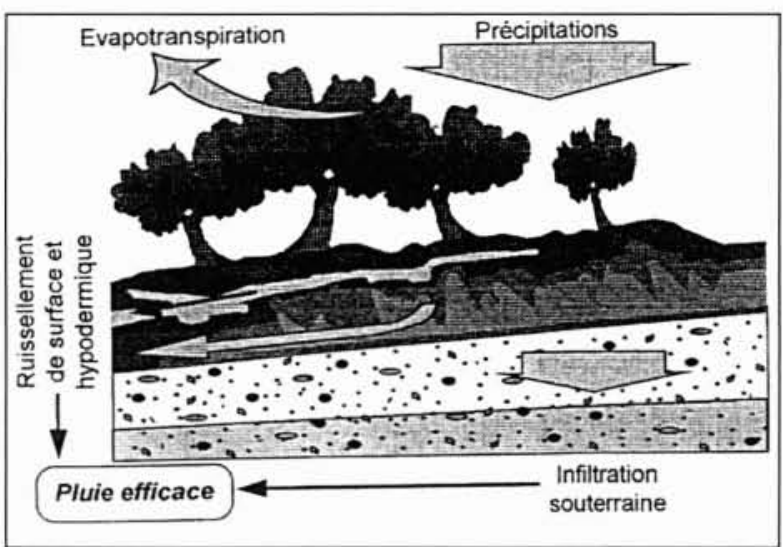

7. Représentation schématique du concept de pluie efficace [19].

\section{II — L'EAU, VECTEUR DE TRANSFERT DES POLLUANTS}

L'eau qui s'infiltre dans le sous-sol permet le transfert d'un certain nombre de composés vers les nappes, essentiellement sous forme dissoute mais potentiellement aussi colloïdale, voire particulaire dans certains cas. Ces quantités d'eau qui s'infiltrent ne représentent qu'une part de la pluviométrie et ne sont pas simplement proportionnelles aux précipitations. En effet, la part qui s'infiltre et qui est susceptible de contribuer à la recharge des nappes est très variable suivant les époques de l'année (température, ensoleillement...), suivant le type de sols et leur couverture, mais aussi selon les caractéristiques mêmes des événements pluvieux (intensité, durée, succession...).

\subsection{L'infiltration}

Comme l'illustre schématiquement la figure 7, l'eau qui s'infiltre dans le sous-sol n'est qu'une part des précipitations, desquelles sont déduites la quantité stockée dans le sol (réserve en eau du sol), l'évapotranspiration (eau évaporée physiquement ou prélevée par la végétation) et le ruissellement. La somme de ce qui s'infiltre en profondeur et de ce qui ruisselle constitue la pluie efficace et contribue à l'alimentation des ressources en eau, qu'elles soient superficielles ou souterraines.

\subsection{Influence de l'infiltration sur les niveaux de contamination des nappes}

La connaissance des quantités d'eau qui s'infiltrent est très importante non seulement pour l'évaluation de la recharge en eau des eaux souterraines, mais également en termes de transfert de polluants.

En France, les apports d'eau aux nappes se situent, selon la nature et le contexte climatique des nappes, entre 50000 et $1000000 \mathrm{de} \mathrm{m}^{3} / \mathrm{an} / \mathrm{km}^{2}$ [18]. Cette variabilité de la recharge a deux conséquences essentielles pour le transfert de polluants. Pour les délais de contamination des nappes, de manière très simplifiée, plus les quantités infiltrées sont grandes, plus les transferts vers la nappe sont rapides (toutes choses égales par ailleurs, notamment la nature physique des terrains traversés par l'eau avant d'atteindre le réservoir souterrain). Par contre, et toujours de manière théorique très simplifiée, les concentrations en solutés seront inversement proportionnelles aux quantités d'eau infiltrées.

A titre d'exemple, en s'appuyant sur une infiltration annuelle moyenne de $200 \mathrm{~mm}$ d'eau par an (bassin Parisien par exemple) ou de $600 \mathrm{~mm}$ (sud-ouest de la France), et pour un reliquat azoté minéral annuel de $20 \mathrm{~kg}$ d'azote $(\mathrm{N})$ par ha, les concentrations en nitrates à la sortie du système sol et susceptibles d'alimenter la nappe seraient respectivement de l'ordre de 45 et $15 \mathrm{mg} / \mathrm{l}$ (concentrations exprimées en $\mathrm{NO}_{3}$ ). Il est clair que la disponibilité des nitrates ou des phytosanitaires pour l'entraînement par les eaux d'infiltration n'est pas distribuée de manière homogène au cours de l'année, et que ces calculs théoriques n'ont qu'une valeur illustrative des variabilités spatiales potentielles.

\subsection{La variabilité temporelle de l'infiltration et son influence potentielle sur la pollution des nappes}

En France, la recharge des nappes, qui dépend en premier lieu des conditions climatiques, se produit essentiellement entre mi-octobre et mi-avril, période pendant laquelle les réserves d'eau des sols sont plus ou moins totalement reconstituées et où l'évapotranspiration n'est pas prépondérante. La figure 8 illustre la variabilité temporelle de cette infiltration au cours d'un cycle annuel, et son influence sur le niveau de la nappe.

Cette variabilité temporelle de l'infiltration a une conséquence importante en terme de transfert de polluants. Suivant la présence et la disponibilité plus ou moins fortes d'un stock

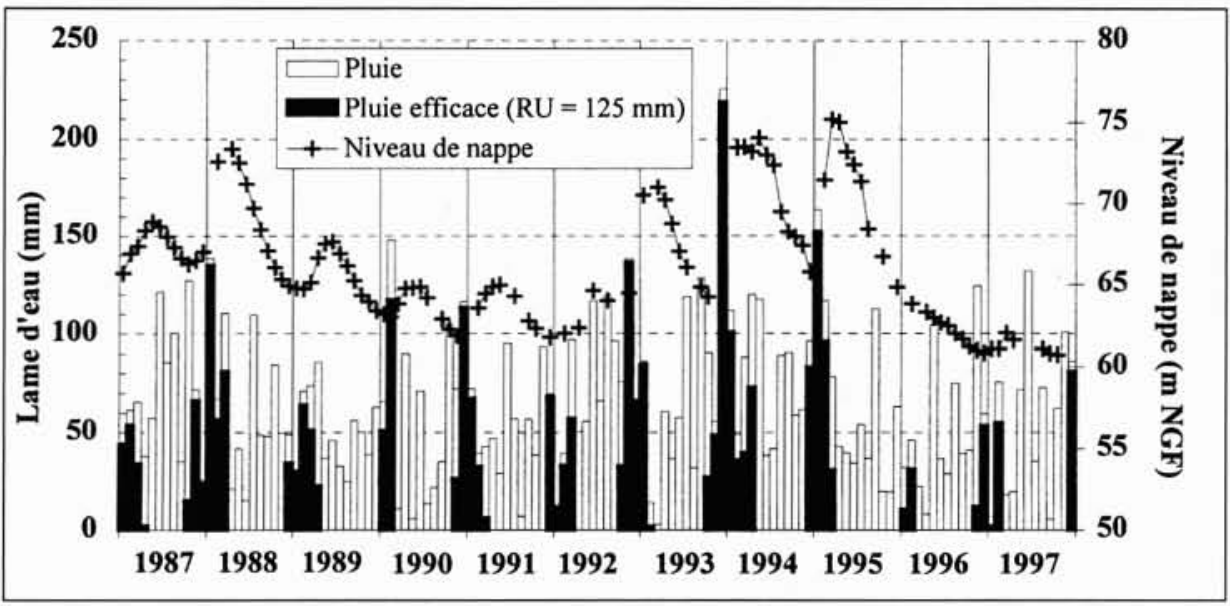

8. Evolution mensuelle entre 1987 et 1997 de la pluie, de la pluie efficace et du niveau de la nappe (bassin versant de l'Hallue, Picardie). 
de polluants à certaines époques de l'année, ces derniers seront plus ou moins entraînés en profondeur. Pour limiter les stocks et la disponibilité des composés pendant la période de recharge des nappes, l'agriculture moderne a mis en place des pratiques " agro-environnementales ". L'attention particulière portée maintenant aux dates d'épandages de produits (fertilisants, pesticides), au fractionnement de ces apports, ainsi que la pratique d'intercultures destinées à immobiliser de manière plus ou moins importante le stock de composés susceptibles d'être entraînés par la recharge vont dans le sens d'une minimisation des risques d'entraînement.

\section{III $\square$ CONCLUSION}

La contamination de certaines nappes par des produits d'origine agricole est une préoccupation majeure pour les consommateurs, les distributeurs d'eau et le monde agricole. La diversité des situations naturelles susceptibles d'être rencontrées, diversité qui n'a pu être que brièvement évoquée ici, rend impossible la formulation de recommandations d'usage applicables de manière générale et dont le succès soit garanti.

Si il est évident que des actions sont nécessaires au niveau des pratiques agricoles, qui doivent être resituées dans la perspective d'un développement durable, une très bonne connaissance du milieu naturel dans sa complexité hydrogéologique est également nécessaire. Par rapport aux eaux superficielles, la complexité des modes de transfert de solutés vers et dans le milieu souterrain rend beaucoup plus ardue la mise en place de plans d'action. Les temps de transfert, en général bien plus longs que dans le domaine superficiel, constituent un écueil supplémentaire à la validation des effets positifs de certains changements éventuels de pratiques agricoles. Dans le cas de molécules potentiellement rémanentes comme les phytosanitaires, et pour les molécules anciennes qui ont été largement utilisées (cas des triazines), de nouvelles pratiques, quelles qu'elles soient, n'auront de toute façon qu'un effet différé sur le retour à des niveaux de concentrations naturels.

Ces difficultés ne doivent toutefois pas bloquer toute velléité d'action. Elles doivent par contre être prises en compte dans les efforts de mise en place d'une agriculture désireuse de concilier des objectifs de rendement avec des souhaits de minimiser les atteintes aux ressources naturelles.

\section{Remerciements}

Cet article est la publication scientifique $n^{\circ} 99010 \mathrm{du}$ BRGM ; il a été réalisé dans le cadre du projet PRR 601 "Pollution diffuse par les phytosanitaires" financé sur crédits de recherche BRGM.

\section{Bibliographie}

[1] TASLI S., 1995. Devenir de l'atrazine en culture de maïs : études en plein champ -site de la côte Saint André, Isère, France- et au laboratoire. Recherche de solutions alternatives de traitement. Thèse de I'Université de Grenoble, 188 pp.

[2] NORMAND B., 1996. Etude expérimentale et modélisation du deveni de l'azote dans le système sol-plante-atmosphère. Thèse de l'Université de Grenoble, 190 pp.

[3] DABENE E., MARIÉ F. et SMITH C., 1995. Substances actives phytosanitaires. Caractéristiques utiles pour l'évaluation du comportement de quelques substances actives dans l'environnement. Ministère de l'Agriculture, de la Pêche et de l'Alimentation. 37p. + 135 fiches.

[4] NORMAND B. et MOUVET C., 1998. Modélisation du transfert préférentiel de l'isoproturon au travers de lysimètres de laboratoire. Actes du XXVIIIème Congrès du Groupe Français des Pesticides, Ecole des Mines d'Alès, sous presse.

[5] STARR J.L. et GLOTFELTY D.E., 1990. Atrazine and bromide movement through a silt loam soil. Journal of Environmental Quality, 19. 552-558.

[6] RICE R.C., JAYNES D.B. et BOWMAN, R.S., 1991. Preferential flow of solutes and herbicide under irrigated fields. Transactions of the ASAE, 34(3), 914-918.

[7] TRAUB-EBERHARD U., HENSCHEL K.P., KÖRDEL W. et KLEIN W., 1995. Influence of different field sites on pesticide movement into subsurface drains. Pesticide Science, 43, 121-129.

[8] BALKWILL D.L., 1988. Numbers, diversity, and morphological characteristics of aerobic, chemoheterotrophic bacteria in deep subsurface sediments from a site in South Carolina. Geomicrobiology Journal, vo 7. 33-52.

[9] DICTOR M.C., 1994. Caractérisation de la distribution et du comportement métabolique de la microflore indigène dans un profil de sol. Thèse INPL-ENSAIA, Nancy, France, 110 p.

[10] LAVY T.L., ROETH F.W. et FENSTER C.R., 1973. Degradation of 2,4 D and atrazine at three soil depths in the field. Journal of Environmental Quality, vol.2, 132-137.

[11] MOORMAN T.B. et HARPER S.S., 1989. Transformation and mineralization of metribuzin in surface and subsurface horizons of a Mississipi Delta soil. Journal of Environmental Quality, vol 18, 302-306.

[12] RODRIGUEZ C.J. et HARKIN J.M., 1997. Degradation of atrazine in subsoils, and groundwater mixed with aquifer sediments. Bulletin of Environmental Contamination Toxicology, vol 59, 728-735.

[13] SEGUIN J.J., 1986. Migration des nitrates dans la zone non saturée de la craie de Champagne. Rapport BRGM 86 SGN 172 EAU.

[14] LANDREAU A. et SEGUIN J.J., 1987. Investigations sur la dénitrification naturelle dans l'aquifere captif de la craie du nord de la France. Rapport BRGM 87 SGN 486 EAU.

[15] PAUWELS H., KLOPPMANN W., FOUCHER J.C., MARTELAT A. Fritsche V., 1998. Field tracer test for denitrification in a pyrite-bearing schist aquifer. Applied Geochemistry (sous presse).

[16] PAUWELS H., LEGENDRE O. et FOUCHER J.C., 1998. High-rate denitrification from several electron donors in a shist aquifer. Proceeding of the 9th International Symposium on Water Rock Interactions, Taupo, New Zealand, 30 March-3 April 1998, Balkema, pp.173-176.

[17] THIERRY D. et SEGUIN J.J., 1986. Un modèle hydrologique global de transfert de nitrates dans un bassin versant. Hydrogéologie, $\mathrm{n}^{\circ} 1,1986$ 87-99.

[18] BODELLE J. et MARGAT J., 1980. L'eau souterraine en France. Ed. Masson.

[19] DAUM J.R., DESPRATS J.F. et DURAND F., 1996. Précipitations efficaces moyennes en France (1965-1994). Rapport BRGM R 38975. 